\title{
THE QUADRATIC COMPLETE INTERSECTIONS ASSOCIATED WITH THE ACTION OF THE SYMMETRIC GROUP
}

\author{
TADAHITO HARIMA, AKIHITO WACHI AND JUNZO WATANABE
}

\begin{abstract}
We prove that any quadratic complete intersection with a certain action of the symmetric group has the strong Lefschetz property over a field of characteristic zero. Furthermore, we discuss under what conditions its ring of invariants by a Young subgroup is a homogeneous complete intersection with a standard grading.
\end{abstract}

\section{Introduction}

It seems natural to conjecture that all (Artinian) complete intersections with standard grading have the strong Lefschetz property over a field of characteristic zero. If there is a group action on a complete intersection, it sometimes enables us to prove that the ring has the property (see [5, Chapter 4]). For example, consider the monomial complete intersection:

$$
A=K\left[x_{1}, x_{2}, \ldots, x_{r}\right] /\left(x_{1}^{n_{1}+1}, x_{2}^{n_{2}+1}, \ldots, x_{r}^{n_{r}+1}\right) .
$$

In spite of the simple nature of the assertion of the strong Lefschetz property, the proof it has the strong Lefschetz property is complicated. If $n_{1}=n_{2}=$ $\cdots=n_{r}=1$, however, Ikeda's lemma provides an easy proof ([6, Lemma 1.1], [5, Proposition 3.67, Corollary 3.70]). It seems remarkable that any monomial complete intersection appears as a subring of the quadratic monomial complete intersection. In fact, the algebra $A$ above is the invariant subring

Received February 23, 2015; received in final form November 2, 2015.

The first author was supported by Grant-in-Aid for Scientific Research (C) (23540052)(15K04812).

The second author was supported by Grant-in-Aid for Scientific Research (C) (23540179).

The third author was supported by Grant-in-Aid for Scientific Research (C) (23540050). 2010 Mathematics Subject Classification. Primary 13E10. Secondary 13F20, 20B35. 
of the quadratic complete intersection $K\left[x_{1}, x_{2}, \ldots, x_{n}\right] /\left(x_{1}^{2}, x_{2}^{2}, \ldots, x_{n}^{2}\right)$ under the group action of the Young subgroup

$$
S_{n_{1}} \times S_{n_{2}} \times \cdots \times S_{n_{r}} \subset S_{n}
$$

where

$$
n=n_{1}+n_{2}+\cdots+n_{r} .
$$

Once we know the strong Lefschetz property in the quadratic case, the general case then follows almost immediately.

The purpose of this paper is to generalize this argument. First, we construct a flat family of quadratic complete intersections, with four parameters, on which the Young subgroup acts in the same way as it does on the quadratic monomial complete intersection. It will be proved that any member in this family has the strong Lefschetz property. It is important to assume that the generators of the defining ideal are quadrics and the symmetric group acts on it. Then we prove that the ring of invariants of any complete intersection in this family by the action of any Young subgroup in $S_{n}$ is again a complete intersection with the strong Lefschetz property. The proof is easy, but the invariant subrings basically do not always have a standard grading. Rather surprisingly, however, it turns out that most of them have the standard grading thanks to the assumption that the generators are quadrics.

The main results of this paper are Theorem 8 and Theorem 9 which are stated in Section 4 and Section 5, respectively. A theorem of Goto says that the ring of invariants of a complete intersection is again a complete intersection if the group is generated by pseudo-reflections and its order is invertible in the ground field. We need to construct a set of uniform generators for all invariant subrings in the family. This is treated in the Appendix.

For simplicity, we will be assuming, mostly, that the characteristic of the ground field $K$ is zero except in Section 2. This is necessary for Facts 6 and Flat Extension Theorem used in the proof of Theorem 8. In the Appendix, we continue to assume $\operatorname{char}(K)=0$, since it is necessary to use the equality $(R / I)^{G}=R^{G} /\left(I \cap R^{G}\right)$.

\section{Definitions}

Definition 1. Let $V=\bigoplus_{i=0}^{\infty} V_{i}$ be a finite dimensional graded vector space and let $L \in \operatorname{End}_{\mathbf{g r}}(V)$ be a graded endomorphism

$$
L: V \rightarrow V
$$

of degree one. Namely a graded endomorphism of degree one is a collection of homomorphisms $\left\{L_{i}: V_{i} \rightarrow V_{i+1}\right\}$. We call $L$ a weak Lefschetz endomorphism if the map $L$ has piece-wise full rank, that is, the restricted map $L_{i}: V_{i} \rightarrow V_{i+1}$ is either injective or surjective for all $i=0,1,2, \ldots$. We will write $L_{i}: V_{i} \rightarrow V_{i+1}$ 
simply as $L: V_{i} \rightarrow V_{i+1}$. We say that $L$ is a strong Lefschetz endomorphism if there exists an integer $c$ such that $V_{i}=0$ for all $i \geq c+1$ and the map $L^{c-2 i}$ restricted to the homogeneous part $L^{c-2 i}: V_{i} \rightarrow V_{c-i}$ is bijective for all $i=0,1,2, \ldots,[c / 2]$. The map $i \mapsto \operatorname{dim}_{K} V_{i}$ is called the Hilbert function of $V$. Sometimes it is written as the power series $\sum_{i=0}^{\infty}\left(\operatorname{dim}_{K} V_{i}\right) T^{i}$. Since $V$ is a finite dimensional vector space, the Hilbert series of $V$ is actually a polynomial in $T$. If a graded homomorphism of $L \in \operatorname{End}_{\mathbf{g r}}(V)$ is a strong Lefschetz endomorphism, it automatically implies that the Hilbert function of $V$ is symmetric about the half integer $c / 2$, where $c=a+b, a$ is the initial and $b$ the end degrees of $V=\bigoplus_{i} V_{i}$.

Lemma 2. Suppose that $V=\bigoplus_{i} V_{i}$ is a finite dimensional graded vector space and that $V$ has a constant Hilbert function. Suppose that $L \in \operatorname{End}_{\mathbf{g r}}(V)$ is a graded endomorphism of degree one. If $L$ is a weak Lefschetz endomorphism, then $L$ is a strong Lefschetz endomorphism.

Proof. Let $a$ be the initial and $b$ the end degrees of $V$, and let $c=a+b$. Then obviously the map $L^{c-2 i}: V_{i} \rightarrow V_{c-i}$ is a bijection for any $i \leq[c / 2]$.

Definition 3. Let $A=\bigoplus_{i=0}^{c} A_{i}$ be a graded (not necessarily standard graded) Artinian $K$-algebra over a field with $A_{0}=K$. We say that $A$ has the weak (resp. strong) Lefschetz property, if there exists a linear form $l \in A_{1}$ such that the multiplication map $L=\times l \in \operatorname{End}_{\text {gr }}(A)$ is a weak (resp. strong) Lefschetz endomorphism. Such a linear form $l$ is called a weak (resp. strong) Lefschetz element. Sometimes we use the abbreviation: WLP (resp. SLP) for weak (resp. strong) Lefschetz property.

Definition 4. Let $A=\bigoplus_{i=0}^{c} A_{i}$ be a graded Artinian $K$-algebra over a field with $A_{0}=K$. The Sperner number of $A$ is defined by

$$
\text { Sperner } A=\operatorname{Max}_{i}\left\{\operatorname{dim}_{K} A_{i}\right\} \text {. }
$$

Proposition 5 (Subring Theorem). Let $A=\bigoplus_{i=0}^{c} A_{i}$ be a graded Artinian $K$-algebra with the strong Lefschetz property. Assume that $A_{c} \neq 0$. Suppose that $B$ is a graded $K$-subalgebra of $A$, that $B_{c}=A_{c}$, and $B_{1}$ contains a strong Lefschetz element for $A$. Then if $B$ has a symmetric Hilbert function, $B$ has the strong Lefschetz property.

Proof. Let $l \in B_{1}$ be a strong Lefschetz element for $A$. Consider the diagram:

$$
\begin{aligned}
A_{i} & \rightarrow A_{c-i} \\
\uparrow & \uparrow \\
B_{i} & \rightarrow B_{c-i},
\end{aligned}
$$

where the vertical arrows are natural injections and horizontal arrows are the multiplication map by $l^{c-2 i}$. The strong Lefschetz property of $A$ implies that $\times l^{c-2 i}: B_{i} \rightarrow B_{c-i}$ is injective. Since $\operatorname{dim}_{K}\left(B_{i}\right)=\operatorname{dim}_{K}\left(B_{c-i}\right)$, it is bijective. 


\section{The polynomial ring and the action of the symmetric group}

Let $R=K\left[x_{1}, x_{2}, \ldots, x_{n}\right]$ be the polynomial ring over $K$, a field of characteristic zero, and let $S_{n}$ be the symmetric group. The homogeneous part of $R$ of degree $d$ is denoted by $R_{d}$. We let the symmetric group $S_{n}$ act on $R$ by permutation of the variables. An element $\sigma \in S_{n}$ is a bijection of the set $\{1,2, \ldots, n\}$. Thus $\sigma$ induces the automorphism of the $K$-algebra $R$ by

$$
f^{\sigma}\left(x_{1}, x_{2}, \ldots, x_{n}\right)=f\left(x_{\sigma(1)}, x_{\sigma(2)}, \ldots, x_{\sigma(n)}\right) .
$$

We recall some basic facts on the representation of $S_{n}$ and its action on $R$ and fix some notation.

FACTS 6.

(i) The irreducible representations of $S_{n}$ are parametrized by the Young diagrams of $n$ boxes. A Young diagram of $n$ boxes is denoted by a partition $\lambda \vdash n$, which is a non-decreasing sequence of positive integers $\lambda=\left(\lambda_{1}, \lambda_{2}, \ldots, \lambda_{k}\right)$ such that $\sum \lambda_{i}=n$.

(ii) We will denote by $V^{\lambda}$ the irreducible module (uniquely determined up to isomorphism) corresponding to $\lambda$. The dimension of $V^{\lambda}$ is determined by the hook length formula. (See, e.g., [7], [9].) Mostly we are interested in partitions of $n$ with at most two rows. Such partitions will be denoted as

$$
(n, 0),(n-1,1), \ldots,(n-[n / 2],[n / 2]) .
$$

Note that $(n, 0)$ denotes the partition with one row.

(iii) Let $U$ be a finite dimensional $S_{n}$-module. The vector space $U$ decomposes as $U=\bigoplus_{\lambda \vdash n} U_{\lambda}$, where $U_{\lambda}$ is a sum of copies of $V^{\lambda}$. The number of times the irreducible module $V^{\lambda}$ occurs in $U$ is the multiplicity of $V^{\lambda}$. Such a decomposition of $U$ is unique up to order of the factors. In other words if $U=\bigoplus_{\lambda \vdash n} U_{\lambda}=\bigoplus_{\lambda \vdash n} U_{\lambda}^{\prime}$, then $U_{\lambda}=U_{\lambda}^{\prime}$ (as vector subspaces of $U$ ) for all $\lambda \vdash n$. Such a decomposition is called the isotypic decomposition of $U$.

(iv) We denote by $Y^{\lambda}$ the Young symmetrizer corresponding to $\lambda \vdash n$. For the meaning of Young symmetrizers, we refer the reader to [7] or [9]. In the sequel all we have to know about $Y^{\lambda}$ is that it gives the projection onto the $\lambda$ isotypical summand

$$
Y^{\lambda}: U \rightarrow U_{\lambda},
$$

for any $S_{n}$-module $U$, where $U=\bigoplus_{\lambda} U_{\lambda}$ is the isotypic decomposition. For example, if $\lambda=(n, 0)$, then $Y^{\lambda}(R)$ coincides with the $\operatorname{ring} R^{S_{n}}$ of invariants of $R$ under the action of $S_{n}$ and $Y^{\lambda}$ is the usual averaging homomorphism. It is well known that $R^{S_{n}}$ is, as a $K$-algebra, generated by the elementary symmetric polynomials. The elementary symmetric polynomial of degree $d$ will be denoted by $e_{d}$. Thus, we have

$$
Y^{(n, 0)}(R)=R^{S_{n}}=K\left[e_{1}, e_{2}, \ldots, e_{n}\right] .
$$


(v) The degree one part $R_{1}$ of $R$ decomposes, as an $S_{n}$-module, as

$$
R_{1} \cong V^{(n, 0)} \oplus V^{(n-1,1)} .
$$

Typical bases for these modules are:

$$
\begin{gathered}
\left\langle x_{1}+x_{2}+\cdots+x_{n}\right\rangle \text { for } V^{(n, 0)}, \\
\left\langle x_{1}-x_{2}, x_{1}-x_{3}, \ldots, x_{1}-x_{n}\right\rangle \text { for } V^{(n-1,1)} .
\end{gathered}
$$

(vi) The degree two part $R_{2}$ of $R$ decomposes, as an $S_{n}$-module, as

$$
R_{2} \cong V^{(n, 0)} \oplus V^{(n, 0)} \oplus V^{(n-1,1)} \oplus V^{(n-1,1)} \oplus V^{(n-2,2)} .
$$

For $V^{(n, 0)}$ we can choose $\left\langle e_{1}^{2}\right\rangle$ and $\left\langle e_{2}\right\rangle$ as bases.

For $V^{(n-1,1)}$ we can choose $\left\langle x_{1}^{2}-x_{2}^{2}, x_{1}^{2}-x_{3}^{2}, \ldots, x_{1}^{2}-x_{n}^{2}\right\rangle$ and $\left\langle\left(x_{1}-x_{2}\right) e_{1},\left(x_{1}-x_{3}\right) e_{1}, \ldots,\left(x_{1}-x_{n}\right) e_{1}\right\rangle$ as bases.

A typical basis for $V^{(n-2,2)}$ is the set of Specht polynomials of shape $(n-2,2)$ :

$$
\left\{\left(x_{1}-x_{j}\right)\left(x_{2}-x_{k}\right) \mid 3 \leq j<k \leq n\right\} \cup\left\{\left(x_{1}-x_{2}\right)\left(x_{3}-x_{k}\right) \mid 4 \leq k \leq n\right\} .
$$

Facts (v) and (vi) can be derived from Molien-Solomon's theorem (e.g., [10, Theorem 9.3.5]), but in this particular case for $R_{1}$ and $R_{2}$ these are easy to see once one knows that the set of Specht polynomials is a basis for $V^{\lambda}$ for any $\lambda \vdash n$. For the definition of Specht polynomials, see [5, Section 9.3].

(vii) By the hook length formula, we have

$$
\operatorname{dim} V^{(n-i, i)}=\left(\begin{array}{c}
n \\
i
\end{array}\right)-\left(\begin{array}{c}
n \\
i-1
\end{array}\right) .
$$

In particular,

$$
\operatorname{dim} V^{(n-2,2)}=\left(\begin{array}{l}
n \\
2
\end{array}\right)-\left(\begin{array}{l}
n \\
1
\end{array}\right)=\frac{n(n-3)}{2},
$$

and

$$
\operatorname{dim} R_{2}= \begin{cases}2 \operatorname{dim} V^{(n, 0)}+2 \operatorname{dim} V^{(n-1,1)}+\operatorname{dim} V^{(n-2,2)}, & \text { if } n>3, \\ 2 \operatorname{dim} V^{(n, 0)}+2 \operatorname{dim} V^{(n-1,1)}, & \text { if } n=3, \\ 2 \operatorname{dim} V^{(n, 0)}+\operatorname{dim} V^{(n-1,1)}, & \text { if } n=2 .\end{cases}
$$

LEMMA 7. With the same notation as above, suppose that $U \subset R_{1} e_{1}$ is a one dimensional $S_{n}$-module. If $n \geq 3$, then $U$ is spanned by $e_{1}^{2}$. If $n=2$, then $U$ is spanned either by $e_{1}^{2}=e_{1}\left(x_{1}+x_{2}\right)$ or $e_{1}\left(x_{1}-x_{2}\right)$.

Proof. Note that $R_{1} \cong R_{1} e_{1}$ as an $S_{n}$-module. Since $R_{1}=Y^{(n, 0)}\left(R_{1}\right) \oplus$ $Y^{(n-1,1)}\left(R_{1}\right)$ is the isotypic decomposition, and $\operatorname{dim} Y^{(n-1,1)}\left(R_{1}\right)=1$ if $n=2$ and $\operatorname{dim} Y^{(n-1,1)}\left(R_{1}\right)>1$ if $n \geq 3$. Thus, the assertion follows. 


\section{Main result}

As in the previous section $R=K\left[x_{1}, x_{2}, \ldots, x_{n}\right]$ denotes the polynomial ring over a field $K$ of characteristic zero. We are interested in the sequences of homogeneous polynomials $f_{i} \in R$ which satisfy the following conditions:

(1) $f_{1}, f_{2}, \ldots, f_{n}$ are quadrics.

(2) $f_{1}, f_{2}, \ldots, f_{n}$ form a regular sequence.

(3) For any $\sigma \in S_{n}$,

$$
f_{i}\left(x_{\sigma(1)}, x_{\sigma(2)}, \ldots, x_{\sigma(n)}\right)=f_{\sigma(i)}\left(x_{1}, x_{2}, \ldots, x_{n}\right) \text { for } i=1,2, \ldots, n .
$$

REMARK 1. If $\left(f_{1}, f_{2}, \ldots, f_{n}\right)$ is a sequence of quadrics in $R$ with the third property above, the stabilizer of $f_{1}$ must be the subgroup $S_{n-1}$ of $S_{n}$ which fixes 1 . Hence, the element $f_{1}$ has the from

$$
p_{0} x_{1}^{2}+p_{1}\left(x_{2}+x_{3}+\cdots+x_{n}\right) x_{1}+p_{2}\left(x_{2}^{2}+x_{3}^{2}+\cdots+x_{n}^{2}\right)+p_{3}\left(\sum_{2 \leq i<j \leq n} x_{i} x_{j}\right),
$$

with four parameters $p_{k}$ and the elements $f_{i}$ are obtained by cyclically permuting the variables. For such a sequence to be a regular sequence, it is necessary and sufficient that the resultant does not vanish. We refer the interested reader to [1] for details on the resultants of homogeneous forms.

Example 1. Put $R=K[x, y, z], e=x+y+z, f=(e-a x)(e-b x), g=$ $(e-a y)(e-b y), h=(e-a z)(e-b z)$. Assume that the resultant of $f, g, h$

$$
a^{5} b^{5}(a-3)(b-3)(a b-a-2 b)^{3}(a b-2 a-b)^{3} \neq 0 .
$$

Then the sequence

$$
f, g, h
$$

satisfy the conditions (1), (2) and (3) of the first paragraph of this section.

TheOREM 8. Assume that the characteristic of $K$ is zero. Let $I=$ $\left(f_{1}, \ldots, f_{n}\right)$ be a complete intersection ideal in $R$ which satisfies the conditions (1), (2) and (3) above. Then $A:=R / I$ has the strong Lefschetz property. Moreover, let $e_{1}=\sum x_{i}$. If $e_{1}^{2} \notin I$, then $e_{1}$ is a strong Lefschetz element for $A$.

Proof. If $e_{1}^{2} \in I$, we can choose $e_{1}^{2}$ as a generator of the ideal $I$. Let $B=$ $K[z] /\left(z^{2}\right)$, with a new variable $z$, and define the map $B \rightarrow A$ by $z \mapsto e_{1}$. It is easy to see that $B \rightarrow A$ is a flat extension and the fiber, say $C$, is the algebra

$$
C=K\left[x_{1}, x_{2}, \ldots, x_{n}\right] /\left(e_{1}, f_{1}, f_{2}, \ldots, f_{n}\right) .
$$

For $i \geq 2$, let $f_{i}^{\prime}$ be the polynomial obtained from $f_{i}$ by the substitution

$$
x_{1} \mapsto-\left(x_{2}+x_{3}+\cdots+x_{n}\right) .
$$

It is easy to see that

$$
C \cong K\left[x_{2}, x_{3}, \ldots, x_{n}\right] /\left(f_{2}^{\prime}, f_{3}^{\prime}, \ldots, f_{n}^{\prime}\right)
$$


so if $\sigma \in S_{n}$ fixes 1 , then $\left(f_{i}^{\prime}\right)^{\sigma}=f_{\sigma(i)}^{\prime}$. Hence, we may induct on $n$ to conclude that the fiber has the SLP. By the Flat Extension Theorem ([5, Theorem 4.10]), the ring $A$ has the strong Lefschetz property.

For the rest of proof, we assume that $I$ does not contain $e_{1}^{2}$. We want to show that $\left(I \cap e_{1} R\right) \cap R_{2}=0$ if $n \geq 3$. Let $h \in\left(I \cap e_{1} R\right) \cap R_{2}$. Since $I$ is generated by a regular sequence, any two linearly independent elements in $I \cap R_{2}$ are not contained in a principal ideal. This implies that $\left(I \cap e_{1} R\right) \cap R_{2}$ is at most one dimensional. If $\sigma \in S_{n}$, it forces $h^{\sigma} R=h R$. In other words, $h$ is a semi-invariant. By Lemma 7, the element $h$ is a scalar multiple of $e_{1}^{2}$ if $n \geq 3$. Since we have assumed that $I$ does not contain $e_{1}^{2}$, we have $h=0$. If $n=2$, then $h \neq 0$ can occur but the assumption $e_{1}^{2} \notin I$ implies that $e_{1}$ is a strong Lefschetz element for $A$.

From now on we assume that $n \geq 3$. Then the sum $R_{1} e_{1}+\left(I \cap R_{2}\right)$ is a direct sum and it contains two copies of $V^{(n, 0)}$ and two copies of $V^{(n-1,1)}$, since both of $R_{1} e_{1}$ and $\left(I \cap R_{2}\right)$ are equivalent to $V^{(n, 0)} \oplus V^{(n-1,1)}$. On the other hand by Facts $6(\mathrm{v})$ and (vi), $R_{1} e_{1}+\left(\left(x_{1}^{2}, \ldots, x_{n}^{2}\right) \cap R_{2}\right)$ also contains two copies of $V^{(n, 0)}$ and two copies of $V^{(n-1,1)}$. By Facts 6(iii), we see that

$$
R_{1} e_{1}+\left(I \cap R_{2}\right)=R_{1} e_{1}+\left(\left(x_{1}^{2}, \ldots, x_{n}^{2}\right) \cap R_{2}\right),
$$

and

$$
I+e_{1} R=\left(x_{1}^{2}, x_{2}^{2}, \ldots, x_{n}^{2}, e_{1}\right) .
$$

In particular, the ideal $I+e_{1} R$ contains all the second powers of the variables. Put $B=R /\left(x_{1}^{2}, x_{2}^{2}, \ldots, x_{n}^{2}\right)$. Generally, it is the case that $\operatorname{dim}_{K}\left(A / e_{1} A\right) \geq$ Sperner $(A)$. (See the proof of [5, Proposition 3.5].) Thus, we have

$$
\operatorname{dim}_{K}\left(B / e_{1} B\right)=\operatorname{dim}_{K}\left(A / e_{1} A\right) \geq \operatorname{Sperner}(A)=\operatorname{Sperner}(B),
$$

and since $B$ has the weak Lefschetz property ([5, Corollary 3.69]), this implies that $\operatorname{dim}_{K}\left(A / e_{1} A\right)=\operatorname{Sperner}(A)$. Hence, $A$ has the weak Lefschetz property.

We have to prove that $A$ has the strong Lefschetz property with $e_{1}$ as an SL element. Let $J=\left(x_{1}^{2}, x_{2}^{2}, \ldots, x_{n}^{2}\right)$. Put $\lambda_{i}=(n-i, i)$ for $i=0,1,2, \ldots,[n / 2]$. Since the way $S_{n}$ acts on $R / J$ and $R / I$ are the same, $A$ and $B$ are isomorphic as $S_{n}$-modules. So we may apply [5, Theorem 9.9] to $A$. Since the $S_{n}$-module $A$ decomposes as

$$
A=\bigoplus_{i=0}^{[n / 2]} Y^{\lambda_{i}}(A)
$$

and since the multiplication map $\times e_{1}: A \rightarrow A$ decomposes as the sum of the restricted maps

$$
\times e_{1}: Y^{\lambda_{i}}(A) \rightarrow Y^{\lambda_{i}}(A), \quad i=0,1, \ldots,[n / 2],
$$


it suffices to prove that the endomorphism $\times e_{1}: Y^{\lambda_{i}}(A) \rightarrow Y^{\lambda_{i}}(A)$ is a strong Lefschetz element for each $i$. Recall that $Y^{\lambda_{i}}(A)$ has a constant Hilbert function ([5, Lemma 9.8]), namely its Hilbert function is

$$
\left(\operatorname{dim} V^{(n-i, i)}\right)\left(T^{i}+T^{i+1}+\cdots+T^{n-i}\right) .
$$

Thus, $A$ has the SLP by Lemma 2 .

\section{Some consequences}

Throughout this section, $K$ denotes a field of characteristic zero as before. Recall that a grading of a graded algebra $A=\bigoplus_{i \geq 0} A_{i}$ is standard if the algebra $A$ is generated by elements of degree one over $A_{0}$. So far we have tacitly assumed that the grading for the algebras $R$ and $A$ are standard. In this section, we consider graded subalgebras which are not necessarily standard graded. We continue to assume that the polynomial ring $R$ has the standard grading, that is, the degrees of the variables are one, but the invariant subrings $R^{G}$ and $A^{G}$ most likely do not have the standard grading. We are primarily concerned however with the cases where the invariant subrings $A^{G}$ do have the standard grading.

ThEOREM 9. Let $A=K\left[x_{1}, x_{2}, \ldots, x_{n}\right] /\left(f_{1}, f_{2}, \ldots, f_{n}\right)$ be a quadratic complete intersection with the action of $S_{n}$ as in Theorem 8. As in Remark 1 we use the notation

$$
\begin{aligned}
f_{1}= & p_{0} x_{1}^{2}+p_{1}\left(x_{2}+x_{3}+\cdots+x_{n}\right) x_{1}+p_{2}\left(x_{2}^{2}+x_{3}^{2}+\cdots+x_{n}^{2}\right) \\
& +p_{3}\left(\sum_{2 \leq i<j \leq n} x_{i} x_{j}\right) .
\end{aligned}
$$

Let $X=\left\{x_{1}, x_{2}, \ldots, x_{n}\right\}$ be the set of variables and let $X=\bigsqcup_{i=1}^{r} X_{i}$ be a partition of the set of variables into $r$ nonempty subsets. Put $n_{i}=\left|X_{i}\right|$ and let

$$
G=S_{n_{1}} \times S_{n_{2}} \times \cdots \times S_{n_{r}}
$$

be the Young subgroup of $S_{n}$ which acts on $R$ in such a way that $S_{n_{k}}$ permutes the variables in the block $X_{k}$ and leaves fixed the variables in other blocks. Assume that $e_{1}^{2} \notin I$. Then $R^{G} /\left(I \cap R^{G}\right)$ is a complete intersection with the strong Lefschetz property. Furthermore, let $S=K\left[y_{1}, y_{2}, \ldots, y_{r}\right]$ be the polynomial ring in $r$ variables and let

$$
\phi: S \rightarrow A
$$

be the homomorphism defined by $\phi\left(y_{i}\right)=\sum_{x \in X_{i}} x$. Then the image $\phi(S)$ coincides with $A^{G}$ for any $\left(p_{0}, p_{1}, p_{2}, p_{3}\right)$ in a nonempty open set in the projective space $\mathbb{P}^{3}=\left\{\left(p_{0}, p_{1}, p_{2}, p_{3}\right)\right\}$. In particular $A^{G}$ has the standard grading if parameters are general enough. 
Proof. The Young subgroup $G \subset S_{n}$ is generated by reflections. By a theorem of Goto [2], the ring of invariants $A^{G}=(R / I)^{G}=R^{G} /\left(I \cap R^{G}\right)$ is a complete intersection. Note that $e_{1} \in A^{G}$ and $e_{1}$ is an SL element for $A$. Moreover, the image of the Jacobian determinant $\left|\frac{\partial f_{i}}{\partial x_{j}}\right|$ in $A$ can be taken as a socle generator for both $A$ and $A^{G}$. By Proposition 5, the $\operatorname{ring} A^{G}$ has the strong Lefschetz property if $e_{1} \in A$ is a strong Lefschetz element. This is a proof for the first assertion of this theorem. For the second assertion, we prove Lemma 10 first.

Lemma 10. Let $Q$ be a Noetherian integral domain, which may contain a field of positive characteristic. Suppose that $R=\bigoplus_{i \geq 0} R_{i}$ is a graded $Q$ algebra, finitely generated over $R_{0}=Q$. (We assume that the graded pieces $R_{i}$ are free $Q$-modules.) If for some maximal ideal $\mathfrak{m}_{0} \subset Q$, the fiber $R / \mathfrak{m}_{0} R:=$ $R \otimes_{Q} Q / \mathfrak{m}_{0}$ has a standard grading, then there exists an ideal $\mathfrak{a} \neq 0$ in $Q$ such that $R / \mathfrak{p} R$ has the standard grading for all prime ideals $\mathfrak{p} \not \supset \mathfrak{a}$.

Proof. Let $Y=\left\{Y_{1}, Y_{2}, \ldots, Y_{r}\right\}$ be a set of homogeneous elements in $R$ such that $Y$ generates the algebra: $R=Q\left[Y_{1}, Y_{2}, \ldots, Y_{r}\right]$. Let $M^{\prime}, M^{\prime \prime}$ be the $Q$-submodules of $R$ as follows: $M^{\prime}=\sum_{i_{1}+i_{2}+\cdots+i_{r} \geq 1} Q Y_{1}^{i_{1}} Y_{2}^{i_{2}} \cdots Y_{r}^{i_{r}}$, and $M^{\prime \prime}=\sum_{i_{1}+i_{2}+\cdots+i_{r} \geq 2} Q Y_{1}^{i_{1}} Y_{2}^{i_{2}} \cdots Y_{r}^{i_{r}}$. Furthermore, put $M=M^{\prime} / M^{\prime \prime}$. Note that $M$ is a graded $Q$-module and $M \otimes_{Q} Q / \mathfrak{m}$ is the tangent space of $R \otimes_{Q} Q_{\mathfrak{m}}$ for any maximal ideal $\mathfrak{m} \subset Q$. It is easy to see that the fiber $R \otimes_{Q} Q / \mathfrak{m}$ has the standard grading if and only if $M \otimes_{Q} Q / \mathfrak{m}$ is spanned by the homogeneous elements of degree one. Let $N$ be the $Q$-submodule of $M$ generated by the degree one elements and put $\mathfrak{a}=\operatorname{Ann}_{Q}(M / N)$. Recall that $\mathfrak{p} \supset \mathfrak{a} \Leftrightarrow(M / N)_{\mathfrak{p}} \neq$ 0 for a prime ideal $\mathfrak{p}$ in $Q$. (See [8] The paragraph preceding Theorem 4.4.) Since there exists at least one maximal ideal such that $M \otimes_{Q} Q / \mathfrak{m}$ has the standard grading, we have $\mathfrak{a} \neq 0$. Then it is straightforward that $\mathfrak{a}$ has the desired property.

Proof of the second part of Theorem 9. Define the polynomials $F$ and $F_{i}$ by

$$
F=P_{0} x_{1}^{2}+P_{1}\left(\sum_{j=2}^{n} x_{j}\right) x_{1}+P_{2}\left(\sum_{j=2}^{n} x_{j}^{2}\right)+P_{3}\left(\sum_{2 \leq k<l \leq n} x_{k} x_{l}\right),
$$

and $F_{i}=F^{\sigma^{i-1}}, i=1,2, \ldots, n$, where $P_{0}, \ldots, P_{3}$ are indeterminates and $\sigma=$ $(12 \cdots n)$ is the cycle of length $n$. These are considered as polynomials in the variables $x_{1}, x_{2}, \ldots, x_{n}$ with coefficients in $K\left[P_{0}, P_{1}, P_{2}, P_{3}\right]$. Let $\mathcal{R}$ be the resultant of $F_{1}, F_{2}, \ldots, F_{n}$. Put $Q=K\left[P_{0}, P_{1}, P_{2}, P_{3}, \mathcal{R}^{-1}\right]$ and we consider the algebra $Q\left[x_{1}, \ldots, x_{n}\right] /\left(F_{1}, \ldots, F_{n}\right)$. In the decomposition of the variables $X=$ $\bigsqcup_{i=1}^{r} X_{i}$ we may assume that the blocks $X_{i}$ consist of variables of consecutive indices. So we assume that the $i$ th block $X_{i}$ is

$$
X_{i}=\left\{x_{n_{1}+\cdots+n_{i-1}+1}, \ldots, x_{n_{1}+\cdots+n_{i}}\right\} .
$$


The numbering of the variables may be illustrated as follows:

$$
\underbrace{x_{1}, \ldots, x_{n_{1}}}_{n_{1}}, \underbrace{x_{n_{1}+1}, \ldots, x_{n_{1}+n_{2}}}_{n_{2}}, x_{n_{1}+n_{2}+1}, \ldots, \underbrace{x_{n-n_{r}+1}, \ldots, x_{n}}_{n_{r}} .
$$

For the sake of notation, we rename the variables as:

$$
x_{i j}=x_{n_{1}+n_{2}+\cdots+n_{i-1}+j},
$$

so $x_{i j}$ is the $j$ th variable in the $i$ th block. Suppose that $X_{0}$ is a set of variables. Then by $e_{d}\left(X_{0}\right)$ we denoted the elementary symmetric polynomial of degree $d$ in the variables in $X_{0}$.

Introduce a set of new variables $Y_{i j}$ of degree one which are indexed as follows:

(1) The first index $i$ ranges $i=1,2, \ldots, r$.

(2) The second index $j$ ranges, depending on $i$, over $j=1,2, \ldots, n_{i}$.

Define the polynomials $\left\{E_{i j}\right\}$ with the same indices as the variables $\left\{Y_{i j}\right\}$ as follows:

$$
E_{i d}=e_{d}\left(\left\{Y_{i 1}, Y_{i 2}, \ldots, Y_{i n_{i}}\right\}\right) \quad \text { for } i=1,2, \ldots, r, d=1,2, \ldots, n_{i} .
$$

Define the algebras $\Lambda$ and $\Lambda^{\prime}$ by

$$
\begin{aligned}
\Lambda & =Q\left[\left\{Y_{i j}\right\}\right] /\left(F_{1}, \ldots, F_{n}\right), \\
\Lambda^{\prime} & =Q\left[\left\{E_{i j}\right\}\right] /\left(\left(F_{1}, \ldots, F_{n}\right) \cap Q\left[\left\{E_{i j}\right\}\right] .\right.
\end{aligned}
$$

Note that $\Lambda$ is mapped onto $A$ by the specialization $P_{k} \mapsto p_{k}, Y_{i j} \mapsto x_{i j}$. By Corollary 12 in the Appendix, it is possible to write

$$
\left(F_{1}, \ldots, F_{n}\right) \cap Q\left[\left\{E_{i j}\right\}\right]=\left(F_{1}^{\prime}, F_{2}^{\prime}, \ldots, F_{n}^{\prime}\right) .
$$

(Note that $F_{1}^{\prime}, \ldots, F_{n}^{\prime}$ are constructed from $F_{1}, \ldots, F_{n}$ explicitly.) The algebra $\Lambda^{\prime}$ is a flat extension of $Q=K\left[P_{0}, P_{1}, P_{2}, P_{3}, \mathcal{R}^{-1}\right]$ and each fiber coincides with $A^{G}$ under the map $Y_{i j} \mapsto x_{i j}$.

On the other hand the image of $\phi: S \rightarrow A$ is a subring of $A^{G}$ and they coincide if and only if $A^{G}$ has the standard grading or equivalently $A^{G}$ is generated by degree one elements. For $P_{0}=1, P_{1}=P_{2}=P_{3}=0$, it is easy to see that the fiber has the standard grading (cf. [5, Lemma 3.70]). Thus, we may apply Lemma 10. This completes the proof of Theorem 9.

REMARK 2. Let $\Lambda$ be the algebra defined in the last paragraph of the proof of the second part of Theorem 9. The fiber for $p_{0}=1, p_{1}=p_{2}=p_{3}=0$ is isomorphic to the monomial complete intersection

$$
K\left[y_{1}, y_{2}, \ldots, y_{r}\right] /\left(y_{1}^{n_{1}+1}, y_{2}^{n_{2}+1}, \ldots, y_{r}^{n_{r}+1}\right) .
$$

This is proved if $r=2$ in [5, Lemma 3.70]. The same proof in fact works for all $r$. 
The following example shows that a member can fail to have the SLP in a flat family of Artinian algebras whose general members have the SLP. It also shows that the embedding dimension is not a constant in a flat family of Artinian algebras.

EXAMPLE 2. Let $p, x, y$ be variables and consider $K[p, x, y] /\left(y^{2}, x^{3}-p y\right)$. We regard it as a family of Artinian algebras. Give the variables $p, x, y$ degrees $0,1,3$ respectively. For any $p \in K$, the Hilbert function of $R$ is

$$
\frac{\left(1-T^{3}\right)\left(1-T^{6}\right)}{(1-T)\left(1-T^{3}\right)}=\frac{\left(1-T^{3}\right)}{(1-T)}\left(1+T^{3}\right)=\frac{1-T^{6}}{1-T}=1+T+\cdots+T^{5} .
$$

If $p=0$, the fiber is $K[x, y] /\left(x^{3}, y^{2}\right)$ and if $p \neq 0$, then it is $K[x] /\left(x^{6}\right)$.

The following example illustrates Theorem 9.

Example 3. Consider the family of the Artinian algebras

$$
K\left[p_{0}, p_{1}, p_{2}, p_{3}\right][v, w, x, y, z] /\left(f_{1}, f_{2}, f_{3}, f_{4}, f_{5}\right),
$$

on which $S_{5}$ acts by the permutation of the variables $\{v, w, x, y, z\}$ and $f_{i}^{\sigma}=$ $f_{\sigma(i)}$ for $\sigma \in S_{5}$. We will use the same notation as the first paragraph of Section 4, and Remark 1 with $n=5$, so

$$
\begin{aligned}
f_{1}= & p_{0} v^{2}+p_{1}(w+x+y+z) v+p_{2}\left(w^{2}+x^{2}+y^{2}+z^{2}\right) \\
& +p_{3}(w x+w y+w z+x y+x z+y z) .
\end{aligned}
$$

Other generators $f_{2}, \ldots, f_{5}$ are obtained by permuting the variables. Consider the Young subgroup

$$
G:=S_{2} \times S_{3},
$$

which acts on $A$ with the division of the variables:

$$
\{v, w, x, y, z\}=\{v, w\} \sqcup\{x, y, z\} .
$$

Then the ring $A^{G}$ of invariants has the Hilbert function

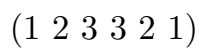

and most cases it is generated by degree one elements. But for $p_{0}=5, p_{1}=2$, $p_{2}=0, p_{3}=2$, the algebra $K\left[\left(A^{G}\right)_{1}\right] \subset A^{G}$ has the Hilbert function

Some more such examples are:

$$
\begin{aligned}
& \left(p_{0}, p_{1}, p_{2}, p_{3}\right)=(0,0,3,8), \\
& \left(p_{0}, p_{1}, p_{2}, p_{3}\right)=(7,7,3,8), \\
& \left(p_{0}, p_{1}, p_{2}, p_{3}\right)=(4,3,2,6), \\
& \left(p_{0}, p_{1}, p_{2}, p_{3}\right)=(6,0,0,4), \\
& \left(p_{0}, p_{1}, p_{2}, p_{3}\right)=(6,3,0,2), \\
& \left(p_{0}, p_{1}, p_{2}, p_{3}\right)=(1,1,3,8) .
\end{aligned}
$$


This example as well as Example 4 below were computed by the computer algebra system Macaulay2 [3].

If $A=R / I$ is not a quadratic complete intersection, then in the general case the ring of invariants of $A$ does not have the standard grading. In the next example, we exhibit such a case.

ExAmple 4 . Let $R=\mathbb{Q}\left[x_{1}, x_{2}, \ldots, x_{6}\right], I=\left(f_{1}, \ldots, f_{6}\right)$, where $f_{i}=x_{i}^{3}$ for $i=1, \ldots, 6$. Let $X_{1}=\left\{x_{1}, x_{2}, x_{3}\right\}$ and $X_{2}=\left\{x_{4}, x_{5}, x_{6}\right\}$ and let $G=S_{3} \times S_{3}$ act on $A=R / I$ by permuting the variables within the blocks $X_{1}$ and $X_{2}$ in the way as described in Theorem 9. Then the ring of invariants $A^{G}$ is, as a $K$-algebra, generated by the six elements of degrees $\{1,2,3,1,2,3\}$ as follows: $r=x_{1}+x_{2}+x_{3}, s=x_{1} x_{2}+x_{1} x_{3}+x_{2} x_{3}, t=x_{1} x_{2} x_{3}, u=x_{4}+x_{5}+x_{6}$, $v=x_{4} x_{5}+x_{4} x_{6}+x_{5} x_{6}$ and $w=x_{4} x_{5} x_{6}$. They satisfy the relations

(1) $u^{3}-3 u v+3 w=0$

(2) $r^{3}-3 r s+3 t=0$,

(3) $u^{2} v-2 v^{2}-u w=0$,

(4) $r^{2} s-2 s^{2}-r t=0$,

(5) $u^{2} w-2 v w=0$

(6) $r^{2} t-2 s t=0$.

Note that the ring $A^{G}$ has in fact embedding dimension 4 and $t$ and $w$ can be eliminated but the grading is not standard. The Hilbert polynomial is

$$
\begin{aligned}
1+ & 2 T+5 T^{2}+8 T^{3}+12 T^{4}+14 T^{5}+16 T^{6}+14 T^{7}+12 T^{8} \\
& +8 T^{9}+5 T^{10}+2 T^{11}+T^{12} \\
= & \left(\left(1+T^{2}\right)\left(1+T+T^{2}+T^{3}+T^{4}\right)\right)^{2} .
\end{aligned}
$$

\section{Appendix}

Proposition 11. Let $R=K\left[x_{1}, \ldots, x_{n}\right]$ be the polynomial ring over a field $K$ of characteristic zero, on which the symmetric group $S_{n}$ acts by permuting the variables. Let $f_{1}, \ldots, f_{n} \in R$ be a set of homogeneous elements which satisfies $f_{i}^{\sigma}=f_{\sigma(i)}$ for any $\sigma \in S_{n}$ for $i=1, \ldots, n$. Define the polynomials $g_{1}, g_{2}, \ldots, g_{n}$ by

$$
\left(\begin{array}{c}
g_{1} \\
g_{2} \\
\vdots \\
g_{n}
\end{array}\right)=\left(\begin{array}{cccc}
1 & 1 & \cdots & 1 \\
x_{1} & x_{2} & \cdots & x_{n} \\
x_{1}^{2} & x_{2}^{2} & \cdots & x_{n}^{2} \\
\vdots & \vdots & & \vdots \\
x_{1}^{n-1} & x_{2}^{n-1} & \cdots & x_{n}^{n-1}
\end{array}\right)\left(\begin{array}{c}
f_{1} \\
f_{2} \\
\vdots \\
f_{n}
\end{array}\right) .
$$

Then the ideal $\left(f_{1}, \ldots, f_{n}\right)$ is a complete intersection if and only if $\left(g_{1}, \ldots, g_{n}\right)$ is a complete intersection.

Proof. The "if" part is obvious. Put $I=\left(f_{1}, \ldots, f_{n}\right)$ and assume that $I$ is an ideal of finite colength, that is, $I$ is a complete intersection. We want to 
prove that $g_{1}, \ldots, g_{n}$ generate an ideal of finite colength. For simplicity, we put $G=S_{n}$. It is possible to construct a minimal free resolution of $\left(f_{1}, \ldots, f_{n}\right)$ which is compatible with the action of $G$. For this, the Koszul complex is enough:

$$
0 \rightarrow \bigwedge^{n} F \rightarrow \cdots \rightarrow \bigwedge^{2} F \rightarrow \bigwedge^{1} F \rightarrow \bigwedge^{0} F
$$

We may think $F$ is the free module generated by $d x_{1}, d x_{2}, \ldots, d x_{n}$, and then extend the action of $G$ to $\bigwedge^{k} F$ (for any $k$ ) in the obvious manner. If $k=1$, it is easy to determine a minimal set of generators for $F^{G}$ as an $R^{G}$-module. This can be done as follows. First, we note that $F^{G}$ is a free $R^{G}$-module of rank $n$. For any $d$, the ideal $\left(x_{1}^{d}, x_{2}^{d}, \ldots, x_{n}^{d}\right) \cap R^{G}$ is generated by the power sum symmetric polynomials of degrees $d, d+1, \ldots, d+n-1$. (This is discussed in the proof of [4, Lemma 7.6].) Hence, it follows that the invariant subspace $F^{G}$ of $F$ is a free $R^{G}$-module of rank $n$ generated by $\left\{\sum_{i=1}^{n} x_{i}^{k} d x_{i} \mid\right.$ $k=0,1, \ldots, n-1\}$. In other words, a matrix $\mathbf{M}$ is determined to be the Van der Monde matrix if it satisfies, for any $d$ given, the following matrix identity:

$$
\left(\begin{array}{c}
x_{1}^{d}+x_{2}^{d}+\cdots+x_{n}^{d} \\
x_{1}^{d+1}+x_{2}^{d+1}+\cdots+x_{n}^{d+1} \\
\vdots \\
x_{1}^{d+n-1}+x_{2}^{d+n-1}+\cdots+x_{n}^{d+n-1}
\end{array}\right)=\mathbf{M}\left(\begin{array}{c}
x_{1}^{d} \\
x_{2}^{d} \\
\vdots \\
x_{n}^{d}
\end{array}\right) .
$$

The first part of the minimal free resolution of $R / I$ takes the form

$$
\bigwedge^{2} F \rightarrow \bigwedge^{1} F \stackrel{\partial}{\rightarrow} R \rightarrow R / I \rightarrow 0
$$

To extract the invariant subspace is an exact functor, so we have the exact sequence

$$
\left(\bigwedge^{2} F\right)^{G} \rightarrow F^{G} \stackrel{\partial}{\rightarrow} R^{G} \rightarrow(R / I)^{G} \rightarrow 0
$$

of free $R^{G}$-modules. The map $\partial: F \rightarrow R$ is defined by

$$
d x_{i} \mapsto f_{i} .
$$

Thus, the image of the restricted map $\partial: F^{G} \rightarrow R^{G}$ is the ideal

$$
\left(g_{1}, g_{2}, \ldots, g_{n}\right) R^{G} \text {. }
$$

This shows that $I \cap R^{G}$ is an ideal of finite colength in $R^{G}$ or equivalently they generate an ideal of finite colength in $R$.

We will call the matrix in the statement of Proposition 11 the Van der Monde matrix. 
Corollary 12. Let $n=n_{1}+\cdots+n_{r}$ be a partition of the integer $n$ and let

$$
\underbrace{x_{1}, \ldots, x_{n_{1}}}_{n_{1}}, \underbrace{x_{n_{1}+1}, \ldots, x_{n_{1}+n_{2}}}_{n_{2}}, x_{n_{1}+n_{2}+1}, \ldots, \underbrace{x_{n-n_{r}+1}, \ldots, x_{n}}_{n_{r}}
$$

be a decomposition of the variables into $r$ blocks. Let $G=S_{n_{1}} \times S_{n_{2}} \times \cdots \times$ $S_{n_{r}} \subset S_{n}$ be a Young subgroup and let $G$ act on $R$ by the block-wise permutation of the variables. Suppose that $f_{1}, \ldots, f_{n}$ is a homogeneous complete intersection which satisfies $f_{i}^{\sigma}=f_{\sigma(i)}, i=1,2, \ldots, n$, for all $\sigma \in G$. Let $V_{i}$ be the Van der Monde matrix in the variables in the ith block

$$
\left\{x_{n_{1}+\cdots+n_{i-1}+j} \mid j=1, \ldots, n_{i}\right\} .
$$

Define the homogeneous elements $g_{1}, g_{2}, \ldots, g_{n}$ by

$$
\left(\begin{array}{c}
g_{1} \\
g_{2} \\
\vdots \\
g_{n}
\end{array}\right)=\left(\begin{array}{cccc}
V_{1} & 0 & \cdots & 0 \\
0 & V_{2} & \cdots & 0 \\
\vdots & \vdots & \ddots & \vdots \\
0 & 0 & \cdots & V_{r}
\end{array}\right)\left(\begin{array}{c}
f_{1} \\
f_{2} \\
\vdots \\
f_{n}
\end{array}\right) .
$$

(The matrix is a block diagonal matrix.) Then

$$
\left(g_{1}, g_{2}, \ldots, g_{n}\right)=\left(f_{1}, f_{2}, \ldots, f_{n}\right) \cap R^{G} .
$$

Proof. $\left(g_{1}, g_{2}, \ldots, g_{n}\right) \subset\left(f_{1}, f_{2}, \ldots, f_{n}\right) \cap R^{G}$ is obvious. Put

$$
\Omega=R d x_{1} \oplus R d x_{2} \oplus \cdots \oplus R d x_{n}, \quad I=\left(f_{1}, f_{2}, \ldots, f_{n}\right) .
$$

Construct a minimal free resolution of $R / I$ over $R$ as:

$$
0 \rightarrow \bigwedge^{n} \Omega \rightarrow \cdots \rightarrow \bigwedge^{1} \Omega \stackrel{\partial}{\rightarrow} R \rightarrow R / I \rightarrow 0
$$

so that the boundary maps are compatible with the action of the group $G$. By taking the invariant subspaces for $G$, we may get the minimal free resolution of $(R / I)^{G}$. As in Proposition 11, the invariant subspace $\Omega^{G}$ as an $R^{G}$-module can be generated by the elements which appear as the entries of the column vector:

$$
\left(\begin{array}{cccc}
V_{1} & 0 & \cdots & 0 \\
0 & V_{2} & \cdots & 0 \\
\vdots & \vdots & \ddots & \vdots \\
0 & 0 & \cdots & V_{r}
\end{array}\right)\left(\begin{array}{c}
d x_{1} \\
d x_{2} \\
\vdots \\
d x_{n}
\end{array}\right)
$$

The map $\Omega \stackrel{\partial}{\rightarrow} R$ is defined as $d x_{i} \mapsto f_{i}$. Hence, we obtain the module $R^{G} /$ $\left(R^{G} \cap\left(f_{1}, \ldots, f_{n}\right)\right)$ as represented by $R^{G} /\left(g_{1}, \ldots, g_{n}\right) R^{G}$. 
REMARK 3. We have been unable to determine a set of generators for $\left(\bigwedge^{k} F\right)^{G}$ for $k>1$ except for $k=n-1, n$. If $k=n$, then $F^{n}$ is the free $R^{G}$-module of rank one with

$$
\left(\prod_{1 \leq k<l \leq n}\left(x_{l}-x_{k}\right)\right) d x_{1} \wedge d x_{2} \wedge \cdots \wedge d x_{n}
$$

as a generator. If $k=n-1$, a set of generators can be specified similarly.

Acknowledgment. The authors would like to thank Larry Smith very much for helpful comments to improve this paper.

\section{REFERENCES}

[1] I. M. Gelfand, M. M. Kapranov and A. V. Zelevinsky, Discriminants, resultants and multidimensional determinants, Birkhäuser, Boston, MA, 1994. MR 1264417

[2] S. Goto, Invariant subrings under the action by a finite group generated by pseudoreflections, Osaka J. Math. 15 (1978), no. 1, 47-50. MR 0480547

[3] D. Grayson and M. Stillman, Macaulay2, available at http://www.math.uiuc.edu/ Macaulay2/.

[4] T. Harima and J. Watanabe, The strong Lefschetz property for Artinian algebras with non-standard grading, J. Algebra 311 (2007), no. 2, 511-537. MR 2314722

[5] T. Harima, T. Maeno, H. Morita, Y. Numata, A. Wachi and J. Watanabe, The Lefschetz properties, Lecture Notes in Mathematics, vol. 2080, Springer-Verlag, Heidelberg, 2013. MR 3112920

[6] H. Ikeda, Results on Dilworth and Ress numbers of Artinian local rings, Japan. J. Math. (N.S.) 22 (1996), no. 1, 147-158. MR 1394376

[7] G. James and A. Kerber, The representation theory of the symmetric group, Encyclopedia of Mathematics and Its Applications, vol. 16, Addison-Weslay, Reading, MA, 1981. MR 0644144

[8] H. Matsumura, Commutative ring theory, Cambridge Studies in Advanced Mathematics, vol. 8, Cambridge University Press, Cambridge, 1989. MR 1011461

[9] B. Sagan, The symmetric group, 2nd ed., Graduate Texts in Mathematics, vol. 203, Springer-Verlag, New York, 2001. MR 1824028

[10] L. Smith, The polynomial invariants of finite groups, Research Notes in Mathematics, vol. 6, A K Peters, Wellesley, MA, 1995. MR 1328644

Tadahito Harima, Department of Mathematics Education, Niigata University, NiIGATA, 950-2181, JAPAN

E-mail address: harima@ed.niigata-u.ac.jp

Akinito Wachi, Department of Mathematics, Hokkaido University of EducaTION, KUSHIRO, 085-8580, JAPAN

E-mail address: wachi.akihito@k.hokkyodai.ac.jp

Junzo Watanabe, Department of Mathematics, Tokai University, Hiratsuka, 259-1292, JAPAN

E-mail address: watanabe.junzo@tokai-u.jp 